Balázs Dombovári, Richárd Fiáth, Bálint Péter Kerekes, Emília Tóth, Lucia Wittner, Domonkos Horváth, Karsten Seidl, Stanislav Herwik, Tom Torfs, Oliver Paul, Patrick Ruther, Herc Neves and István Ulbert*

\title{
In vivo validation of the electronic depth control probes
}

\begin{abstract}
In this article, we evaluated the electrophysiological performance of a novel, high-complexity silicon probe array. This brain-implantable probe implements a dynamically reconfigurable voltage-recording device, coordinating large numbers of electronically switchable recording sites, referred to as electronic depth control (EDC). Our results show the potential of the EDC devices to record good-quality local field potentials, and single- and multiple-unit activities in cortical regions during pharmacologically induced cortical slow wave activity in an animal model.
\end{abstract}

Keywords: electronic depth control; microelectrode probe array; neural recording.

\footnotetext{
*Corresponding author: István Ulbert, Institute of Cognitive Neuroscience and Psychology, Research Centre for Natural Sciences, Hungarian Academy of Sciences, Budapest, Hungary-1025, E-mail: ulbert@cogpsyphy.hu; and Faculty of Information Technology, Pázmány Péter Catholic University, Budapest, Hungary-1083 Balázs Dombovári, Richárd Fiáth, Bálint Péter Kerekes, Emília Tóth, Lucia Wittner and Domonkos Horváth: Faculty of Information Technology, Pázmány Péter Catholic University, Budapest, Hungary; and Institute of Cognitive Neuroscience and Psychology, Research Centre for Natural Sciences, Hungarian Academy of Sciences, Budapest, Hungary

Karsten Seidl, Stanislav Herwik, Oliver Paul and Patrick Ruther: Department of Microsystems Engineering (IMTEK), University of Freiburg, Freiburg, Germany

Tom Torfs: IMEC, Leuven, Belgium

Herc Neves: Department of Engineering Sciences, Uppsala

University, Uppsala, Sweden
}

\section{Introduction}

The scientific need for multiple-site brain electrical activity recording capability fueled the elaboration of various multisite voltage-recording microelectrode probe arrays. Different technological solutions have been reported to implement these devices depending on the user demand, brain area to explore, and experimental approach. Manual construction technologies, such as wire-based probe assembly, were used earlier to build probes and record neural signals with many sites in acute or in chronic experiments. Although these manual technologies are considered to be outdated, there are still many laboratories using hand-assembled stereotrodes [11], tetrodes $[13,21]$, and other micro-wire bundle arrays $[9,10]$. The reason for their continuing usage is mostly because of the convenience of their construction, their durability, and the possibility to physically reconfigure the location of the wire-based probes with respect to the brain tissue, if experimental needs require it.

In the last decades, microelectromechanical systems (MEMS) technology became expedient enough to take the technological lead in constructing brain-implantable devices [22]. As the most commonly used carrier material of these probes is silicon ( $\mathrm{Si}$ ), they are called nowadays the silicon probes [1]. There is a strong demand from the scientific point of view to record from as many locations from the brain as possible to better understand the large-scale machinery of neural networks, facilitate reproducibility, and to characterize interindividual differences. To fulfill the experimental necessities of the mass neural recording demand, usually a limited number (16-32) of recording sites are implemented on a single silicon probe shaft and the probe is physically moved with respect to the brain tissue in order to explore gradually more and more brain areas. Of course, this approach poses the risk of probe breakage, especially in the case of the rigid silicon carrier and, most important, tissue damage due to frictional forces and bleeding from the rupture of blood vessels [6].

To overcome the fragility and tissue damage problem and establish large numbers of neuronal recording sites in a relatively wide brain area, we have developed electronic depth control (EDC) devices using MEMS or complementary metal-oxide semiconductor (CMOS) technology [12, 15, $17,20]$. These devices resemble a regular silicon probe in shape; however, instead of having only a limited number of recording sites, they have 188 recording contacts occupying a considerably larger proportion of the area of their 4-mm long shaft. Of the 188 possible contacts, eight sites can be electronically selected and routed out to an external 
amplifier to record neuronal activity simultaneously from the eight sites. The selected sites can be rapidly reconfigured, allowing the experimenter to record from widespread brain areas without physically moving the device $[18,19]$.

In this study, we used the anesthesia-induced slow wave activity (SWA) model to validate the performance of the EDC probes in cortical structures. We found that our device can record excellent-quality local field potentials (LFP), singleand multiple-unit activity (SUA, MUA), and spectral activity that are all according to the general physiological appearance of the SWA. We conclude that EDC devices are capable of fulfilling the experimental demand aimed toward a versatile probe system that is capable of rapidly reconfigurable, mass neuronal recording while avoiding mechanical damage to the brain due to physical motion.

\section{Methods}

\section{Electronic depth control device}

The 180- $\mu \mathrm{m}$-wide, $80-\mu \mathrm{m}$-thick, 4 -mm-long, $19^{\circ}$ tip angle penetrating shaft of the EDC device was realized using deep reactive ion etching (DRIE) of silicon [17]. The shaft is populated with 188 recording sites in two columns with an equal horizontal and vertical center pitch of 40 $\mu \mathrm{m}$. The $20-\mu \mathrm{m}$-diameter recording sites were plated with Pt, measuring about $1 \mathrm{MOhm}$ impedance at $1 \mathrm{kHz}$. Each recording site can be connected internally to one of the eight output lines of the probe, and these output lines can be connected in turn to an outside amplifier [18, 19]. The connection matrix, switching electronics, control, and input and output lines were integrated on the shaft of the probe, implemented by the 0.6- $\mu \mathrm{m}$ CMOS process XC06 (X-FAB Semiconductor Foundries AG, Erfurt, Germany). Post-CMOS fabrication, including the recording site, metallization and DRIE of the CMOS wafer for electrode shaft shaping were carried out at IMTEK (University of Freiburg, Freiburg, Germany). The input and output lines of the probe were wire bonded to a printed circuit board (PCB) and encapsulated with technical grade epoxy (Figure 1). The EDC device is reconfigurable using a field-programmable gate array (FPGA)-based controller (XC3S200, XILINX Spartan) on a separate PCB, through the parallel port of a personal computer. The graphical user interface (GUI, NeuroSelect) software made it possible to visualize electrode selection and reselect different configurations according to the experimental situation [18]. The settling time after configuration reselection depended on the $\mathrm{DC}$ value mismatch between the

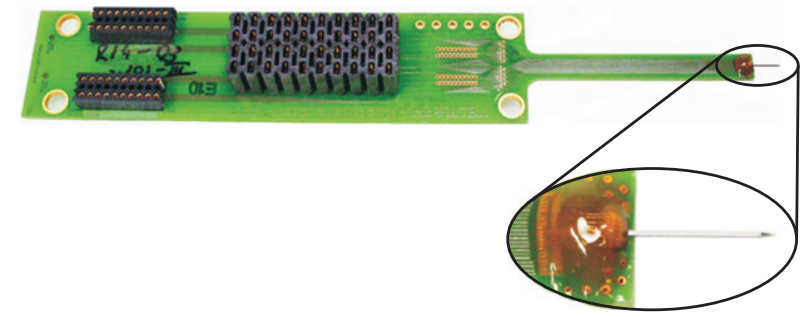

Figure 1 Assembled 4-mm-long active probe shaft and close-up of the probe bonded to a PCB encapsulated by two-component epoxy.

electrodes switched and the time constant of the amplifier used. The switching transient itself lasted about 10-100 ms. The GUI was written using the free multiplatform wxWidgets framework and DialogBlocks, based on $\mathrm{C}++$. Further details about the EDC probe fabrication and GUI and FPGA interfacing can be found elsewhere [17-19].

\section{Implantation procedures and induction of the SWA}

Wistar rats ( $\mathrm{n}=5$, weight of $250-350 \mathrm{~g}$ ) were used for the experiments. All procedures were approved by the Animal Care Committee of the Institute of Cognitive Neuroscience and Psychology, Research Centre for Natural Sciences, Hungarian Academy of Sciences, Budapest, Hungary. Initial anesthesia was administered through intramuscular injection of a mixture of $37.5 \mathrm{mg} / \mathrm{ml}$ ketamine and $5 \mathrm{mg} / \mathrm{ml}$ xylazine at $0.2 \mathrm{ml} / 100 \mathrm{~g}$ body weight; temperature was maintained at $37^{\circ} \mathrm{C}$ throughout the 1-4-h-long recording sessions. The anesthesia was maintained with successive updates of the same drug combination of $0.2 \mathrm{ml} / \mathrm{h}$. Animals were placed in a stereotaxic frame (David Kopf Instruments, Tujunga, CA, USA), and craniotomy was performed over the trunk region of the primary somatosensory cortex (S1) anterior-posterior: (AP: -1.0 -4.0), medial-lateral: (ML: $1.0+4.0$ ), with respect to the bregma [14]. The probe was attached to a manual microdrive (David Kopf Instruments) through its mounting PCB, and it was slowly $(0.1 \mathrm{~mm} / \mathrm{s})$ inserted in the $\mathrm{S} 1$ trunk region AP: $-2.6 \mathrm{~mm}$, ML: $2.5 \mathrm{~mm}$ with respect to the bregma driven by hand. The probes usually penetrated the dura and pia mater without bending, breaking, and causing significant brain dimpling or visible bleeding. After recording from the trunk region of $\mathrm{S} 1$ for $1-4 \mathrm{~h}$, the probe was withdrawn and the animal was sacrificed.

Ketamine/xylazine anesthesia is known to induce a characteristic brain activity called the slow wave activity. The oscillation is roughly in the $\sim \mathrm{Hz}$ range; it is clearly composed of two states, the active "up-state" and the silent "down-state". The up-state LFP is positive on the 
brain surface and negative in deeper layers; it contains nested higher-frequency oscillations (e.g., 5-100 Hz) accompanied by cell firing. The down-state LFP is negative on the brain surface and inverts to positivity in the deeper layers, and contains no spectral or cellular firing activity $[2,3,16]$.

\section{Recording procedures}

Before the implantation, impedance measurements were carried out to confirm the integrity of the probe, using $250 \mathrm{nA}, 1 \mathrm{kHz}$ sine wave as testing signal (EASI-1; BAK Electronics, Mount Airy, MD, USA) injected into selected recording sites. The measurements were done in Ringer's lactate solution (TEVA, Budapest, Hungary) against an $\mathrm{Ag} / \mathrm{AgCl}$ reference electrode. In all cases, the impedances were measured between 0.5 and $1 \mathrm{MOhm}$ for the functioning electrode sites. The impedance measurements were also repeated while the probe was implanted in the brain, with roughly similar results (data not shown).

The outputs of the EDC probe were fed into a highinput impedance referential preamplifier (bandwidth of DC-100 kHz, gain=10). Stainless-steel needle ground and reference electrodes were placed on the left and right side of the craniotomy. Wide bandwidth electrical activity (0.1$7000 \mathrm{~Hz}$ ) with an overall gain of 1000 was recorded (with custom-made filter amplifier) at $20 \mathrm{kHz} /$ channel sampling rate, on eight channels, with 16-bit precision (PCI6259, LabView; National Instruments, Austin, TX, USA) and stored in hard drive. To extract the LFP, the wideband data were further band-pass filtered at $0.1-500 \mathrm{~Hz}$, $24 \mathrm{~dB} /$ oct, zero phase shift. To extract SUA and MUA, the raw data were further band-pass filtered at 500-5000 $\mathrm{Hz}, 24 \mathrm{~dB} /$ oct, zero phase shift offline using NeuroScan Edit 4.3 software (Compumedics, El Paso, TX, USA). The recording site selection was sent to the EDC probe through the FPGA-based controller using the NeuroSelect GUI software [18].

\section{Results}

\section{General remarks}

In the scope of the current study, we investigated the LFP, MUA, SUA, and spectral signatures of the SWA. More specifically, we identified the spatiotemporal and spectral properties of SWA-related LFP and SUA/MUA, and the phase relationship of the LFP and SUA/MUA during SWA, and compared these observations to the existing knowledge to justify the use of the EDC device.

\section{Temporal, spectral, and spatial properties of the SWA}

LFP recordings were conducted with the EDC probe from the trunk region of the primary somatosensory area $(n=8$ penetrations). A representative example of the LFP and MUA depth profile is shown in Figure 2A spanning the whole cortical thickness approximately evenly in Figure $2 \mathrm{~B}$ and $\mathrm{D}$, respectively. The time series of SWA was characterized by the rhythmic recurrence of positive and negative half-waves in the recorded LFP traces (Figure 2B). Close to the surface, up-states (darker gray shading) were characterized by large positive deflections crowned by higher-frequency (spindle and gamma range) LFP oscillations (channels 1-3 in Figure 2B), while in the cortical depth, the up-states were negative, and the trough of the wave was also characterized by higher-frequency

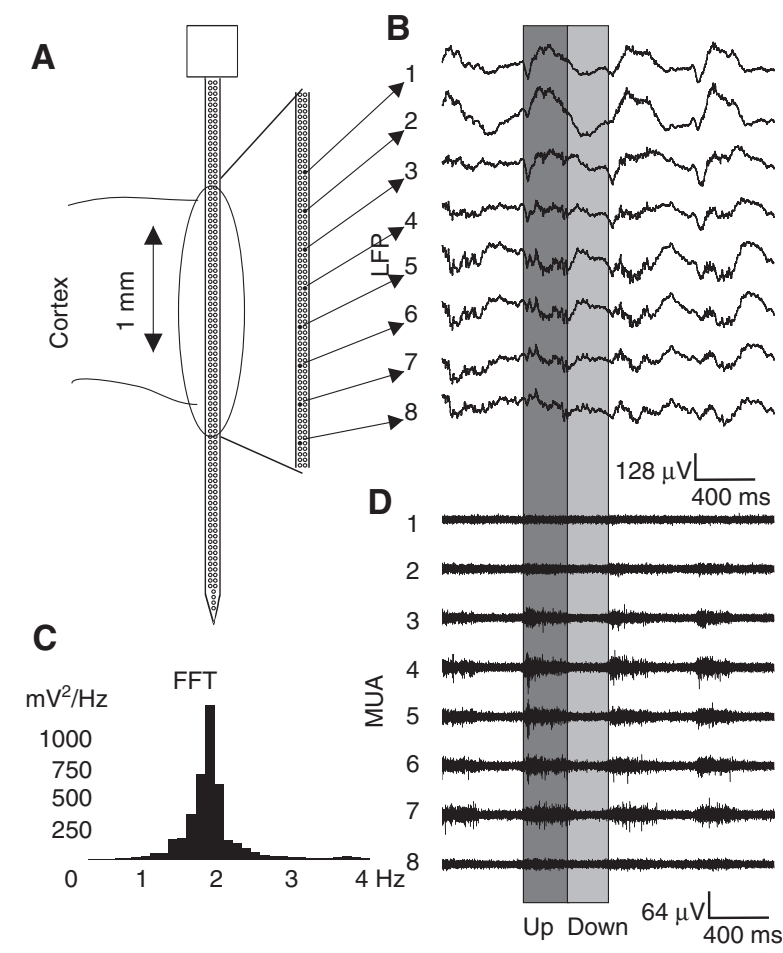

Figure 2 (A) Approximate recording position of the 4-mm long, active probe in the cortex. Close-up of eight roughly equidistant recording locations separated by approximately $300 \mu \mathrm{m}$. (B) Example LFP traces from the eight recording locations. Rhythmically recurring positive (dark gray) and negative (light gray) half-waves are highlighted. (C) FFT of the LFP spectrum. (D) Example MUA traces from the eight recording locations. The original raw data were bandpass filtered between 500 and $5000 \mathrm{~Hz}$. 
oscillations. The down-states (lighter gray shade) were negative in LFP recording close to the cortical surface and inverted into positivity in the deeper layers. Multiple unit firing (Figure 2D) and higher-frequency oscillations were low in all layers of the cortex during down-states. The frequency spectrum of the oscillation was calculated using the fast Fourier transform (FFT) algorithm. The LFP data were cut into 8192-ms-long segments and averaged in the frequency domain using cosine window smoothing. We found that the average peak frequency of the SWA was usually in the $1-2 \mathrm{~Hz}$ range (Figure $2 \mathrm{C}$ ).

As the EDC probe was implanted under the surveillance of a surgical microscope, we were able to verify the depth of the implantation by counting the recording contacts outside of the brain. To evaluate the spatial pattern of the LFP phase inversion, we recorded from eight roughly equidistant locations from the depth of the cortex spreading all layers, separated by approximately $300 \mu \mathrm{m}$. We found a clear LFP phase inversion of the SWA in all of our recordings. The phase inversion was usually located between 300 and $600 \mu \mathrm{m}$ depth measured from the pia mater (Figure 2B, contacts 2-4).

\section{Detection of up- and down-states}

To evaluate the electrophysiological features of the SWA, it is indispensable to analytically define up- and downstates. To obtain an estimate on the timing of the state transitions, we defined the up- and down-state onsets by using MUA measures.

According to previous studies, extracting the envelope of the MUA helps identifying state transitions [3]. To extract the MUA envelope, first the wide band signals were band-pass filtered (500-5000 Hz, $24 \mathrm{~dB} /$ octave, zero phase shift) and rectified (Figure $3 \mathrm{~A}$ ). On the resulting traces, an additional low-pass smoothing filter was applied (30 Hz, $24 \mathrm{~dB}$ /octave, zero phase shift) to obtain the envelope of the signals (Figure 3B). In the next step, the up- to down-state and down- to up-state transitions were defined (Figure 3C). First, we identified the largest up-state/down-state amplitude ratio channel, which was usually located in layer V. Down-states were manually defined, a marker was placed in the deepest trough of the down-state, and the average and standard deviation (SD) of these points were calculated. We defined the state transition thresholds as the average plus two SDs. If two upstates or two down-states were separated by $<100 \mathrm{~ms}$, the two states were extended and merged together.

To test if this threshold measure had a meaningful physiological explanation, the amplitude histogram of
A

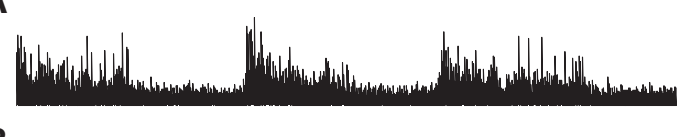

B

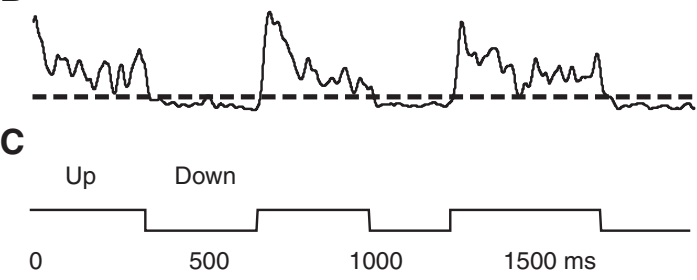

D

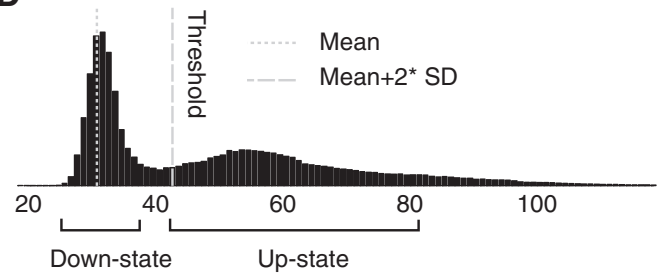

Figure 3 State detection based on MUA.

(A) Example MUA trace obtained from raw data by band-pass filtering $(500-5000 \mathrm{~Hz})$ and rectifying (arbitrary units). (B) Low-pass-filtered $(30 \mathrm{~Hz})$ MUA used for state detection. (C) Result of state detection based on the low-pass-filtered MUA in B. (D) Amplitude histogram of MUA envelope.

the MUA envelope was constructed. In all of the cases, this histogram was found to be bimodal, indicating the presence of two processes, more specifically the up- and down-states of the oscillation (Figure 3D). In addition, the calculated threshold value was always located between the two peaks of the bimodal distribution (Figure 3D, dashed line).

\section{Averaged depth profiles of the SWA}

By using the time stamps of the down- to up-state transition as a reference point, the average spatial, temporal, and spectral patterns of the SWA were calculated to relate our measurements to the findings of other results using different probes $[2,3,16]$.

We found that the up-state locked, averaged LFP profile showed an amplitude inversion at the third contact located around $600 \mu \mathrm{m}$ below the pia (Figure 4A). The corresponding MUA depth profile showed maximal amplitude on the fourth or fifth electrode corresponding to 900$1200 \mu \mathrm{m}$ cortical depth (Figure 4B).

Joint time-frequency analysis was performed on the recorded LFP data using wavelet-based methods [5]. The spectral content of the oscillation was calculated from single sweep LFP waveforms followed by averaging of the resultant individual time-frequency measures. Dividing 

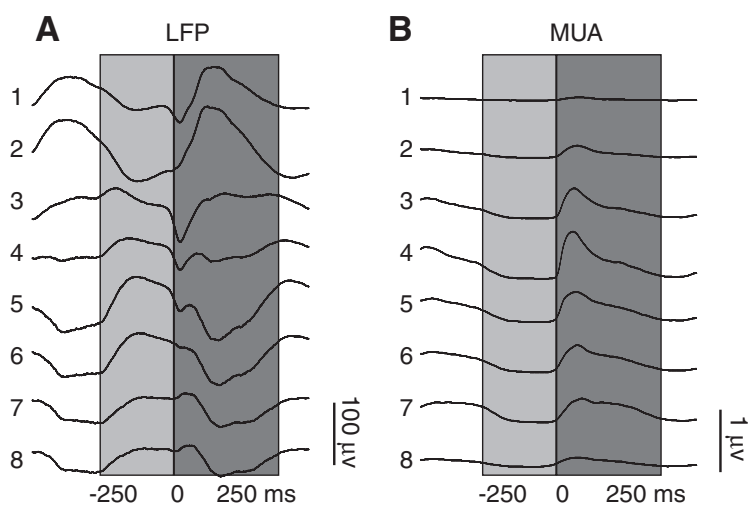

Figure 4 Up-state locked averages.

(A) Up-state locked averaged LFP traces recorded from the eight locations (see close-up in Figure 2A). Amplitude inversion between contacts 2 and 3 corresponding to an approximate recording depth of $600 \mu \mathrm{m}$. (B) Up-state locked averaged MUA traces. Maximal amplitude on contacts 4-5 corresponding to an approximate recording depth of 900-1200 $\mu \mathrm{m}$. Light shading indicates downstate; dark shading indicates up-state.

the wavelet amplitude values with that of a distant baseline $(-1000$ to $-500 \mathrm{~ms})$ in each frequency band gives the relative change of spectral activity in time expressed in $\mathrm{dB}$. We found that up-states were characterized by increased oscillatory activity mainly in the gamma range $(30-80 \mathrm{~Hz})$ in all of the layers, while in the down-state the spectral activity was decreased in all layers (Figure 5).

\section{Properties of SUA}

Putative SUA was analyzed by filtering, threshold detection, and clustering methods using custom-made Matlab software. The wide-band signal was further digitally filtered (500-5000 Hz, zero phase shift, $24 \mathrm{~dB} /$ octave, in Neuroscan Edit 4.3) to eliminate low-frequency contamination of the action potential data (Figure 6A). After threshold recognition [4] at a given channel (mean $\pm 3-5 \mathrm{SD}$, each channel separately), two representative amplitude values (e.g., peak and trough) were assigned to each unclustered action potential waveform. These duplets were projected into the two-dimensional space (Figure 6B), and a competitive expectation-maximization-based algorithm [7] was used for cluster cutting [8] (Figures 6C and D). If the autocorrelogram (Figures $6 \mathrm{E}, \mathrm{F}$, and $\mathrm{G}$ ) of the resulting clusters contained action potentials within the 2-ms refractory interval, it was reclustered. If reclustering did not yield a clean refractory period, the action potential was regarded as originated from multiple cells and omitted from the single cell analysis. We found that the
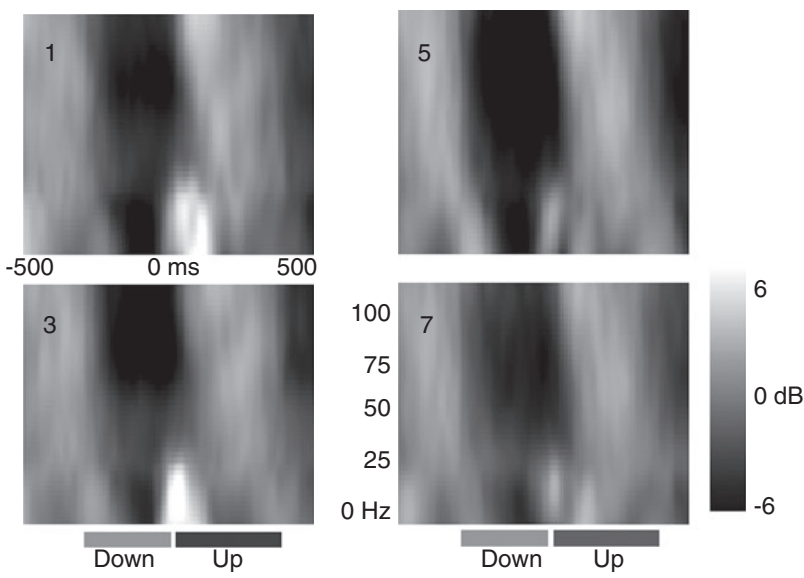

Figure 5 Average time-frequency maps of up-state locked epochs on selected recording channels $1,3,5$, and 7 separated by approximately $600 \mu \mathrm{m}$.

See close-up in Figure 2A for distribution of recording channels (1-7) along the probe shaft. Increased (light colors) oscillatory activity in the gamma range $(30-80 \mathrm{~Hz})$ during up-state and decreased (dark colors) spectral activity during down-state in all layers.

clustered single units exhibit an autocorrelation profile that reveals mostly three main oscillatory processes. Unit bursting at fast time scales $(200 \mathrm{~Hz})$ is visible in Figure 6E, spindle activity $(8-17 \mathrm{~Hz})$ is revealed in Figure $6 \mathrm{~F}$ marked with a single asterisk, while the SWA is pointed out at a longer recurrence rate corresponding to about $1.5 \mathrm{~Hz}$ frequency (Figure 6F, double asterisk). Interaction between two separated single units are visible on the cross-correlogram (Figure $6 \mathrm{H}$ ) revealing about the same $1.5 \mathrm{~Hz}$ recurrence rate as in Figure 6F.

To further characterize the relationship of sorted unit firing and LFP phase, both up- and down-states were individually (sweep by sweep) divided into 10 phase bins and the corresponding sorted single unit firing was depicted on a histogram. The results of such analysis are depicted in Figure 7, where three separated single units are related to the phase of the SWA. We found that most of the firing is happening during the up-state of the SWA, and only a minor activity is taking place in the silent phase.

To test the temporal stability of the recording system, if a putative single unit was found at a given site, the probe was configured using the NeuroSelect software to record from a distant location. After usually 5-10 min, without moving the device, the probe was reconfigured to the location where the single unit was originally found. In all of the attempts $(n=5)$, we were able to find the same putative single unit, proving the reliability of the recording site switching software, hardware, and the stability of the probe within the cortex. 


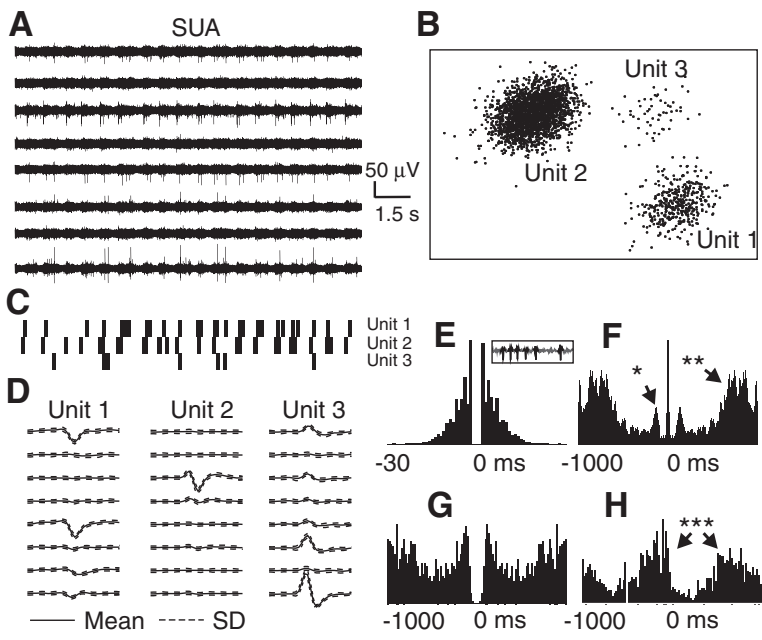

Figure 6 (A) Representative SUA traces. (B) Isolated clusters of three units from A. (C) Raster plots of the three isolated units in B. (D) Mean spike waveforms with SD of the three isolated units in $B$ along the eight recording channels (Figure $2 A$ ). (E) Autocorrelogram of unit 2 firing. Inset: burst firing of unit 2. (F) Autocorrelogram of unit 2 firing with longer time scale. $\left({ }^{*}\right)$ marks spindle modulation and $(* \star)$ marks SWA modulation of unit firing. (G) Autocorrelogram of unit 1 firing. (H) Cross-correlogram of unit 1 and unit 2 firing. ( $\left.{ }^{\star \star \star}\right)$ marks SWA modulation in the cross-correlogram.

\section{Discussion}

Anesthesia-induced SWA consists of the alternation of two states in the membrane potential of neurons: an active state (up-state), during which the membrane potential is closer to the firing threshold and neurons generate action potentials, and a hyperpolarized, silent state (down-state), during which the membrane potential is more negative with virtually no firing. With the aid of the EDC probe, we were able to detect both states of the SWA with high reliability using LFP, MUA/SUA, and spectral measures.
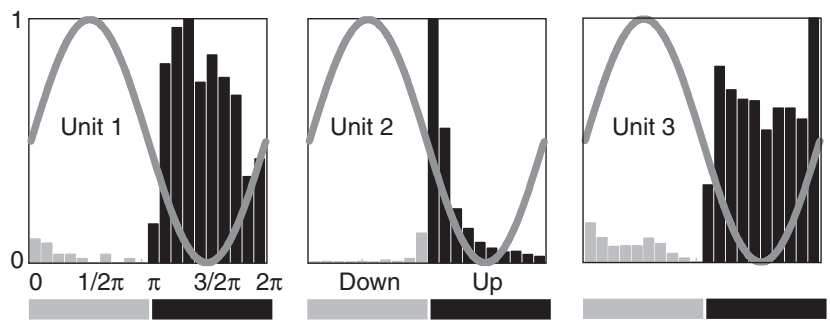

Figure 7 Phase histograms of three representative unit firings related to the phase of SWA.

Phase bins are represented on the horizontal axis and normalized firing rates are represented on the vertical axis. Light bars indicate down-state; dark bars indicate up-state. In all three cases, unit firing mostly occurs during up-state, while during down-state only minor unit activity is observed.
More specifically, we found that the LFP is rhythmic at about $1 \mathrm{~Hz}$; its depth profile well corresponds to previous findings, where the LFP inversion was taking place in layer III corresponding to a depth about 300-500 $\mu \mathrm{m}$ below the cortical surface $[2,3,16]$. We have also shown that the bimodality of the oscillation using MUA measures can be reliably characterized, which is also in correspondence with the basic properties of SWA and previous findings. In addition, similar analysis techniques can be implemented on the EDC data as they were used on data obtained by the classic silicon probes.

Besides LFP and MUA measures, another characterizing feature of SWA is the spectral signature of cortical electrical activity indexed by an LFP spectrogram. Previous investigations showed large cortical oscillatory power in a wide frequency band $(10-200 \mathrm{~Hz})$ during up-states, while the spectral power was much smaller during down-states. Our findings are in a perfect match with these reports $[2,3,16]$. Consistent with prior studies in animals, we have shown that the up-state was associated with increased firing and elevated spindle and gamma power during the surface-positive LFP half-wave, while the down-state was characterized by the widespread surface-negative LFP half-wave with decreased firing, and oscillatory activity.

Single unit clustering ability is another key feature of silicon probes, in general. We found that the EDC probe is capable of recording well-sortable single units, and these clustered cells show similar properties as similarly processed records of classic silicon probes. We have also shown that reliable unit detection and search is possible with the EDC system by just switching between the recording channels rather than moving the device in the brain.

In conclusion, we have shown that the EDC system is an equally capable device to record brain activity as existing silicon probe implementations. In addition to the classic devices, it has the ability to map large areas of cortical fields without being physically moved, which makes this device a unique tool for less invasive neural recordings. Owing to the fast and non-invasive reconfiguration of recording contacts, the EDC device may significantly increase the amount of information that can be obtained during a single experiment as compared with passive silicon probes.

Acknowledgments: NeuroProbes EU FP6, ANR/NKTH Neurogen, ANR/NKTH Multisca, OTKA 81357, OTKA PD77864, TÁMOP-4.2.1.B-11/2/KMR-2011-0002, Bolyai Research Fellowship.

Received May 23, 2013; accepted August 30, 2013 


\section{References}

[1] Campbell PK, Jones KE, Huber RJ, Horch KW, Normann RA. A silicon-based, three-dimensional neural interface: manufacturing processes for an intracortical electrode array. IEEE Trans Biomed Eng 1991; 38: 758-768.

[2] Chauvette S, Volgushev M, Timofeev I. Origin of active states in local neocortical networks during slow sleep oscillation. Cereb Cortex 2010; 20: 2660-2674.

[3] Csercsa R, Dombovári B, Fabó D, et al. Laminar analysis of slow wave activity in humans. Brain 2010; 133 : 2814-2829.

[4] Csicsvari J, Hirase H, Czurko A, Buzsaki G. Reliability and state dependence of pyramidal cell-interneuron synapses in the hippocampus: an ensemble approach in the behaving rat. Neuron 1998; 21: 179-189.

[5] Delorme A, Makeig S. EEGLAB: an open source toolbox for analysis of single trial EEG dynamics including independent component analysis. J Neurosci Methods 2004; 134: 9-21.

[6] Grand L, Wittner L, Herwik S, et al. Short and long term biocompatibility of NeuroProbes silicon probes. J Neurosci Methods 2010; 189: 216-229.

[7] Harris KD, Henze DA, Csicsvari J, Hirase H, Buzsaki G. Accuracy of tetrode spike separation as determined by simultaneous intracellular and extracellular measurements. J Neurophysiol 2000; 84: 401-414.

[8] Heitler JW. DataView v5: software for the display and analysis of digital signals in neurophysiology. 2006.

[9] Karmos G, Molnar M, Csepe V. A new multielectrode for chronic recording of intracortical field potentials in cats. Physiol Behav 1982; 29: 567-571.

[10] Kubie JL. A driveable bundle of microwires for collecting single-unit data from freely-moving rats. Physiol Behav 1984; 32: $115-118$.

[11] McNaughton BL, O’Keefe J, Barnes CA. The stereotrode: a new technique for simultaneous isolation of several single units in the central nervous system from multiple unit records. J Neurosci Methods 1983; 8: 391-397.
[12] Neves HP, Torfs T, Yazicioglu RF, et al. The NeuroProbes project: a concept for electronic depth control. In: 30th International IEEE EMBS Conference, Vancouver, Canada, 2008: 1857

[13] O'Keefe J, Recce ML. Phase relationship between hippocampal place units and the EEG theta rhythm. Hippocampus 1993; 3: 317-330.

[14] Paxinos G, Watson CH. The rat brain in stereotaxic coordinates. San Diego, CA: Academic Press 1998.

[15] Ruther P, Herwik S, Kisban S, Seidl K, Paul O. Recent Progress in Neural Probes Using Silicon MEMS Technology. IEEJ Trans Elec Electron Eng 2010; 5: 505-515.

[16] Sakata S, Harris KD. Laminar structure of spontaneous and sensory-evoked population activity in auditory cortex. Neuron 2008; 64: 404-418.

[17] Seidl K, Herwik S, et al. CMOS-based high-density silicon microprobe arrays for electronic depth control in intracortical neural recording. I microelectromech Syst 2011; 20: 1439-1448.

[18] Seidl K, Torfs T, Mazière PA, et al. Control and data acquisition software for high-density CMOS-based microprobe arrays implementing electronic depth control. Biomed Tech (Berl) 2010; 55: 183-191.

[19] Seidl K, Schwaerzle M, Ulbert I, Neves HP, Paul O, Ruther P. CMOS-based high-density silicon microprobe arrays for electronic depth control in intracortical neural recording characterization and application. IEEE J MicroElectromech Syst 2012; 21: 1426-1435.

[20] Torfs T, Aarts A, Erismis M, et al. Two-dimensional multichannel neural probes with electronic depth control. IEEE Trans Biomed Circ Syst 2011; 5: 403-412.

[21] Wilson MA, McNaughton BL. Dynamics of the hippocampal ensemble code for space. Science 1993; 261: 1055-1058.

[22] Wise KD, Angell JB, Starr A. An integrated-circuit approach to extracellular microelectrodes. IEEE Trans Biomed Eng 1970; 17: 238-247. 